\title{
PRIMORDIAL MOLECULAR HYDROGEN PRODUCTION AND THE FORMATION OF POPULATION III OBJECTS
}

\author{
J. C. N. de Araujo and R. Opher \\ Instituto Astronômico e Geofísico - U.S.P. \\ C.P. 9638, 01065 São Paulo, S.P. - Brasil
}

\begin{abstract}
We study the formation of Population III objects (and stars) taking into account the expansion of the Universe and a series of physical processes relevant to the primordial plasma. In particular, a very important way to cool the first condensations in the Universe is with molecular hydrogen. The minimum mass of the Population III objects formed is $\sim 10^{4} M_{\odot}$. We show also that stars of mass $\gtrsim 50 M_{\odot}$ can be formed from the fragmentation of Population III objects of mass $\sim 10^{6} M_{\odot}$.
\end{abstract}

\section{INTRODUCTION}

The isothermal density perturbations can be nonlinear for subgalactic scales (e.g. Hogan 1978, Lahav 1986, de Araujo \& Opher 1988, 1989) and lead to the formation of Population III objects.

It is possible to produce nonlinear perturbations for scales $M<10^{8} M_{\odot}$ by isocurvature or adiabatic cold dark matter density perturbations (see de Araujo and Opher 1991).

These Population III objects can collapse directly or fragment, forming in this way the first stars (Population III).The cooling caused by the $\mathrm{H}_{2}$ molecules have a very important role in the evolution and formation of Population III objects, as well as in the masses of the fragments (Population III stars).

\section{CALCULATIONS AND DISCUSSION}

In the study of the formation of Population III objects and stars, we performed calculations take into account a series of processes: the photon-drag and cooling due to the background radiation, recombination, photoionization, collisional ionization, Lyman- $\alpha$ cooling and the formation and cooling of $\mathrm{H}_{2}$ molecules.

The $\mathrm{H}_{2}$ molecules have an important contribution to the thermal evolution of the clouds that we study. Several authors have studied the possible ways in which molecular hydrogen can be formed. In our calculations, we take into account the most efficient processes for the production and the destruction of the $\mathrm{H}_{2}$ molecules: 
$H+e^{-} \rightarrow H^{-}+h \nu$ (Hirasawa 1969); $H+H^{-} \rightarrow H_{2}+e^{-}$(Browne \& Dalgarno); $H^{-}+h \nu \rightarrow H+e^{-}$(Matsuda et al 1969); $H^{+}+H^{-} \rightarrow 2 H$ (Peterson et al 1971); $H+H^{+} \rightarrow H_{2}^{+}+h \nu$ (Ramaker \& Peek 1976); $H_{2}^{+}+H \rightarrow H_{2}+H^{+}$(Karpas, Anicich \& Huntress 1979); $H_{2}^{+}+h \nu \rightarrow 2 H^{+}$(Matsuda et al 1969); $H_{2}^{+}+e^{-} \rightarrow 2 H$ ( Giusti-Suzor, Bardsley \& Derkits 1983); $H_{2}+H \rightarrow 3 H$ (Lepp \& Shull 1983).

For the cooling function of the $H_{2}$ molecules we adopted Lepp \& Shull (1983) which gives good results for $100 \mathrm{~K}<T<10^{5} \mathrm{~K}$ and $n>0.1 \mathrm{~cm}^{-3}$.

We begin the calculations at the recombination era $z_{\text {rec }} \sim 1500$ and consider the expansion of the Universe and take $\Omega=0.1$ (density parameter) and $h=1.0$ (Hubble constant in units of $100 \mathrm{~km} \cdot \mathrm{s}^{-1} \cdot M p \mathrm{c}^{-1}$ ).

We take a spectrum of perturbations of the form

$$
\delta=\frac{\delta \rho}{\rho}=\left(M / M_{o}\right)^{\alpha} /\left(1+z_{\text {rec }}\right)
$$

where $M_{\mathrm{o}}$ is the mass scale and $z_{\text {rec }}$ is the redshift at recombination. We take $M_{o}=10^{15} M_{\odot}$ and $\alpha=1 / 3$.

Our calculations show that the minimum mass that can collapse lias $\sim 10^{4} M_{\odot}$.

We study the formation of Population III stars that can be produced by the fragmentation of clouds of mass $M \sim M_{j}$ (Jeans mass at the beginning of the recombination era, $\left.\sim 10^{5-6} M_{\odot}\right)$. We use the fact that a perturbation for $M \ll M_{j}$ that can survive with some residual amplitude, can produce the fragmentation of clouds of mass $M \sim M_{j}$ in the first free fall time scale of $\mathrm{M}$ (see de Araujo \& Opher 1989).

We obtain that the minimum mass that can fragment from the $M_{c}$ cloud is $\sim 50 M_{\odot}$ at $z \sim 181$.

\section{REFERENCES}

Browne, J. C. \& Dalgarno, A. 1969, J. Phys. B, 2,885

de Araujo, J. C. N. \& Opher, R. 1988 , MNRAS, 231, 923

de Araujo, J. C. N. \& Opher, R. 1989 , MNRAS, $\underline{239}, 271$

de Araujo, J. C. N. \& Opher, R. 1991, ApJ (in press)

Giusti-Suzor, A., Bardsley, J. N. \& Derkits, C. 1983, Phys. Rev. A, 28, 682

Hirasawa, T. 1969, Prog. theor. Phys., $\underline{42,} 523$

Hogan, C. 1978, MNRAS, 185,889

Karpas, Z., Anicich, V. \& Huntress, W. T., 1979, J. Chem. Phys., 70, 2877

Lahav, O. 1986, MNRAS, 220, 259

Lepp, S. \& Shull, J. M., 1983, Ap. J., 270, 578

Matsuda, T., Sato, H. \& Takeda, H., 1969, Prog. theo. Phys., 42,219

Peterson, J. R., Abertz, W. H., Moseley, J. T. \& Sheridan, J. R., 1971, Phys. Rev. A, $\underline{3}, 1651$

Ramaker, D. \& Peek, J. 1976, Phys. Rev. A, $\underline{13}, 58$ 